\title{
Introducing the Sleep Disorders Symptom Checklist-25: A Primary Care Friendly and Comprehensive Screener for Sleep Disorders
}

\author{
Karen J. Klingman, PhD, RN ${ }^{1}$, Carla R. Jungquist, PhD, FNP-BC ${ }^{2}$, Michael L. Perlis, $\mathrm{PhD}^{3,4}$ \\ ${ }^{1}$ SUNY Upstate Medical University College of Nursing, Syracuse, NY, USA \\ 2University at Buffalo (SUNY Buffalo) School of Nursing, Buffalo, NY, USA \\ ${ }^{3}$ Department of Psychiatry, University of Pennsylvania, Behavioral Sleep Medicine Program, Philadelphia, PA, USA \\ ${ }^{4}$ University of Pennsylvania School of Nursing, Philadelphia, PA, USA
}

\begin{abstract}
Background and Objective With sleep disorders highly prevalent and associated with poor health outcomes, screening for sleep disorders in primary care could reduce the burden of chronic diseases and costs of health care. Currently, a brief comprehensive primarycare-friendly multiple-sleep-disorders screening instrument is not available. The Sleep Disorders Symptom Checklist (SDS-CL)-17, a singlepage instrument, was developed to screen for six sleep disorders (insomnia, obstructive sleep apnea, restless legs syndrome/periodic limb movement disorder, circadian rhythm sleep-wake disorders, narcolepsy, and parasomnias) and evaluated psychometrically. SDSCL-17 psychometrics are reported. The resulting development of a more comprehensive single-page 25-item instrument, the SDSCL-25, based on validation study results is described. Approaches for clinical use of the SDS-CL-25 are recommended.
\end{abstract}

Methods A cross-sectional study using nested data from two previous research studies ( $\mathrm{n}=395$ sleep clinic referrals and $\mathrm{n}=299 \mathrm{com}-$ munity volunteers) was used. SDS-CL-17 subscale scores and physician diagnoses were analysed using receiver operator characteristic curves. Resulting cut-point scores determined sensitivities/specificities. Study subject interview data were used to assess patient-friendliness of the instrument.

Results Sensitivities/specificities for the diagnosed sleep disorders ranged from 0.64 to 0.88 . Interviewees endorsed the instrument as userfriendly.

Conclusions While the SDS-CL-17 is useful, the SDS-CL-25 assesses for a much larger number of sleep disorders yet retains brevity and is therefore recommended for ongoing clinical use. Psychometric evaluation of the SDS-CL-25 continues.

Sleep Med Res 2017;8(1):17-25

Key Words Sleep, Sleep wake disorders, Primary health care, Preventive health services, Diagnosis.

Received: February 18, 2017

Revised: April 27, 2017

Accepted: May 17, 2017

Correspondence

Karen J. Klingman, PhD, RN

SUNY Upstate Medical University,

College of Nursing, 750 East Adams Street,

Syracuse, NY 13210, USA

Tel +1-585-208-5336

Fax +1-315-464-5168

E-mail klingmak@upstate.edu

\section{INTRODUCTION}

Sleep disorders, if untreated, increase the risk for the development of obesity, diabetes, cardiovascular disease, and depression and negatively impact the course and treatment of these chronic conditions once developed..$^{1-4}$ With the prevalence of sleep disorders at $10-40 \%$ in the general population, ${ }^{5}$ the detection and treatment of sleep disorders is important for the maintenance of wellness and may serve as a means to reduce the onset and burden of chronic diseases and the overall cost of health care. As important as this opportunity for prophylaxis may be, most medical providers do not inquire about their patients' sleep quality or quantity. This may be due, in part, to a lack of training and expertise regarding the assessment and treatment of sleep disorders. This may also be due to the understandable constraints on providers' time in primary care settings. Time constraints could be overcome, however, if a brief, comprehensive and psychometrically sound sleep disorders screening questionnaire were available. If this were the case, then it might be possible to put the assessment of sleep disorders into regular practice in a manner that is akin to what has occurred with depression in the last decade. That is, with the advent of brief screeners for depression, e.g., the nine-item Patient Health Questionnaire (PHQ-9), ${ }^{6,7}$ it became possible to assess for depression in the primary care setting. In the case of sleep disorders, the screening process is complicated by the fact that not one, 
but multiple disorders need to be evaluated.

To date, several attempts have been made to create a comprehensive self-report sleep disorders assessment tool. In a recent review of these instruments, ${ }^{8}$ seven were identified as comprehensive screeners but only one was found to be relatively comprehensive, brief and psychometrically sound: the Global Sleep Assessment Questionnaire (GSAQ). ${ }^{9}$ This instrument assesses for four sleep disorders, excessive daytime sleepiness (EDS) and impaired daytime function and is especially user-friendly for primary care settings because of its single-page layout and grid format with a header design that captures patient characteristics such as work schedule. The GSAQ, however, is not truly comprehensive as it does not assess for circadian rhythm disorders, narcolepsy, nightmare disorder, night terror disorder, rapid eye movement (REM) sleep behavior disorder, or sleeprelated temporomandibular joint disorder (TMJ). Further, it does not provide for an evaluation of initial, middle, or late insomnia. During the early 2000s, and in parallel with the development of the GSAQ, a research screener called the Sleep Disorders Symptom Checklist (SDS-CL)-17 was developed. This instrument covered six categories of sleep disorders in a singlepage grid layout. Recently, this instrument underwent psychometric evaluation to assess its degree of concordance with polysomnography and physician diagnoses. The purpose of the present report is to 1) summarize the findings from this evaluation, 2) describe how these findings led to the development of the SDS-CL-25, and 3) explain the plan for the ongoing validation of the SDS-CL-25.

\section{METHODS}

\section{Study Design and Participants}

This cross-sectional study used nested data of samples from two previous research studies: 1) Who had been referred by primary care providers to a sleep clinic for confirmatory sleep disorder diagnoses $(n=395)^{10}$ and 2) Community volunteer participants in a study that included diagnosing for obstructive sleep apnea (OSA) and insomnia as part of its protocol $(\mathrm{n}=$ 299). ${ }^{11}$ Data for the sleep clinic patients was obtained in New York State and Illinois between 2009-2011 as part of the regular intake procedure for several clinical practices; data were collected in sequence for all incoming patients with no inclusion/exclusion criteria. Data for the community sample were collected in New York State between 2012-2014. The community volunteer study utilized actigraphy, home respiratory sleep tests, questionnaires, and sleep diaries; exclusion criteria were previous sleep disorder diagnosis, or use of continuous positive airway pressure or supplementary oxygen while sleeping.

The use of these data for a validation study was approved by the Institutional Review Board at the State University of New York at Buffalo.

\section{Measures}

\section{Subject characteristics}

Measures collected in both samples $(n=694$ total) were age, sex, height and weight; body mass index (BMI) was calculated from height and weight. For the community sample only, race, ethnicity, and educational level were also collected. All subjects completed the SDS-CL-17 (see below), and were characterized for daytime sleepiness using the Epworth Sleepiness Scale (ESS). ${ }^{12}$ The community volunteers were evaluated for insomnia symptoms using the Insomnia Severity Index (ISI). ${ }^{13}$ Study participants were interviewed about the content and ease-of-use of the SDS-CL-17. The major outcome variables for the study were 1) SDS-CL-17 subscale scores (see below), 2) expert diagnostic assessments for each disorder, 3) receiver operator characteristics (ROC) curve determined thresholds for the six SDS-CL-17 subscale scores, and 4) patient assessments regarding the easeof-use of the SDS-CL-17.

\section{The Sleep Disorders Symptom Checklist-17}

The SDS-CL-17 was developed as a paper instrument to screen potential research study participants for six categories of sleep disorders: insomnia, OSA, restless legs syndrome/periodic limb movement disorder (RLS/PLMD), circadian rhythm sleep-wake disorders, narcolepsy, and parasomnias. The instrument text is laid out as a grid on an $8.5 \times 11$ single sided page and requires about 1-3 minutes for completion. Questions and response options were based on international classification of sleep disorders (ICSD)-2 and DSM-IV diagnostic criteria ${ }^{14}$ and expert opinion. Symptoms are framed as simple statements, e.g." "I am awake 30 or more minutes during the night", with subjects asked to rate how frequently the statement is of relevance for them. The time frame for responses is "over the course of the last year" and the rating scale (response options) ranges from "never" to "frequently (> $3 \mathrm{x} /$ week)". The symptom items and response options are arrayed so that completed questionnaires form a grid of "X" mark patterns depending on the participants' sleep disorder diagnoses. Each of the 17 statements are clustered by disorder (Fig. 1) with 4 items dedicated to insomnia disorder, 4 for OSA, 3 for RLS/PLMD, 2 for circadian rhythm sleep-wake disorders, 2 for narcolepsy, and 3 for parasomnias. Summing the items per subscale adds to 18 (vs. 17) because one of the 17 items is used for both OSA and insomnia. The subscale scores are determined by summing responses in each subscale (Fig. 1, lower portion).

\section{Expert diagnoses}

Sleep disorder diagnoses were obtained for both samples. The sleep clinic diagnoses were determined by a board certified sleep medicine physician based on in-lab polysomnography, questionnaires, and interviews. The community sample diagnoses were provided by a board certified sleep medicine physician 


\begin{tabular}{|c|c|c|c|c|c|c|c|c|}
\hline & \multicolumn{3}{|c|}{ Over the past year: } & Never & $\begin{array}{l}\text { Seldom } \\
(1 \times \mathrm{yr})\end{array}$ & $\begin{array}{l}\text { Sometimes } \\
(1-3 \times \mathrm{mo})\end{array}$ & $\begin{array}{c}\text { Often } \\
(1-3 \times \mathrm{wk})\end{array}$ & $\begin{array}{l}\text { Frequently } \\
(>3 \times w \mathrm{w})\end{array}$ \\
\hline 1 & \multicolumn{3}{|c|}{ In takes me 30 minutes or more to fall asleep. } & & & & & \\
\hline 2 & \multicolumn{3}{|c|}{ I am awake 30 minutes or more during the night. } & & & & & \\
\hline 3 & \multicolumn{3}{|c|}{ I am awake 30 minutes or more prior to my scheduled wake time or alarm. } & & & & & \\
\hline 4 & \multicolumn{3}{|c|}{ I am tired, fatigued or sleepy during the day. } & & & & & \\
\hline 5 & \multicolumn{3}{|c|}{ I sleep better if I go to bed before 9:00 pm and wake up before 5:30 am. } & & & & & \\
\hline $\begin{array}{l}6 \\
7\end{array}$ & \multicolumn{3}{|c|}{$\begin{array}{l}\text { I sleep better if I go to bed late (after 1:00 am) and wake up late (after 9:00 am). } \\
\text { I fall asleep at inappropriate times or places. }\end{array}$} & & & & & \\
\hline 8 & \multicolumn{3}{|c|}{ I have been told that I snore. } & & & & & \\
\hline 9 & \multicolumn{3}{|c|}{ I wake up during the night choking or gasping. } & & & & & \\
\hline 10 & \multicolumn{3}{|c|}{ I have been told I stop breathing when I sleep. } & & & & & \\
\hline 11 & \multicolumn{3}{|c|}{$\begin{array}{l}\text { I feel uncomfortable sensations in my legs, especially when sitting or lying } \\
\text { down that are relieved by moving them. }\end{array}$} & & & & & \\
\hline 12 & \multicolumn{3}{|c|}{ I have an urge to move my legs that is worse in the evenings and nights. } & & & & & \\
\hline 13 & \multicolumn{3}{|c|}{$\begin{array}{l}\text { I have experienced sudden muscle weakness when laughing, joking, angry } \\
\text { or during other intense emotions. }\end{array}$} & & & & & \\
\hline $\begin{array}{l}15 \\
16\end{array}$ & \multicolumn{3}{|c|}{$\begin{array}{l}\text { I have been told that I walk, talk, eat or act strange or violent while sleeping. } \\
\text { I have nightmares. }\end{array}$} & & & & & \\
\hline 17 & \multicolumn{3}{|c|}{ For no reason, I awaken suddenly, startled, and feeling afraid. } & & & & & \\
\hline \multicolumn{9}{|c|}{$\begin{array}{l}\text { This is the SDS-CL- } 17 \text { instrument that is printed on an } 8.5 \times 11 \text { " sheet of paper. The text below shows how to score each item, and the subscale score above which } \\
\text { a positive screen is indicated. Cut-point score determination is described in the methods and results sections, but are included here for completeness. }\end{array}$} \\
\hline \multicolumn{2}{|c|}{ Item choice } & Item score & Subscale & \multicolumn{2}{|c|}{ Items to sum for subscale score } & \multicolumn{2}{|c|}{ Subscale score range } & point score \\
\hline \multicolumn{2}{|c|}{ Never } & 0 & Insomnia & \multicolumn{2}{|c|}{$1,2,3,4$} & \multicolumn{2}{|c|}{$0-12$} & 5 \\
\hline \multicolumn{2}{|c|}{ Seldom } & 0 & Circadian rhythm & 5,6 & & \multicolumn{2}{|l|}{$0-6$} & - \\
\hline \multicolumn{2}{|c|}{ Sometimes } & 1 & Narcolepsy & 7,14 & & \multicolumn{2}{|l|}{$0-6$} & 1 \\
\hline \multirow{3}{*}{\multicolumn{2}{|c|}{$\begin{array}{l}\text { Often } \\
\text { Frequently }\end{array}$}} & 2 & Obstructive sleep apnea & $4,8,9,10$ & & $0-12$ & & 3 \\
\hline & & 3 & Restless legs syndrome & $11,12,13$ & & $0-9$ & & 3 \\
\hline & & & Parasomnias & $15,16,17$ & & $0-9$ & & - \\
\hline
\end{tabular}

Fig. 1. The Sleep Disorders Symptom Checklist (SDS-CL)-17 instrument and scoring guide.

based on actigraphy, home respiratory sleep tests, questionnaires, and sleep diaries. For the community volunteers, a diagnosis of OSA, in general, corresponded to an apnea hypopnea index of 5 or greater, and a diagnosis of insomnia, in general, corresponded to an ISI score of 10 or greater; however, the physician also utilized clinical judgement which may have been informed by actigraphy data, diaries, and questionnaire responses.

Receiver operator characteristic curve determined cut-points

Cut-point scores for each SDS-CL-17 subscale were determined using ROC curve analyses, ${ }^{15,16}$ with cut-points based on the ROC curve inflection. The ROC curve analyses were performed with sleep disorder diagnosis as the state variable and SDS-CL-17 subscale score as the test variable. For each ROC curve, area under the curve (AUC) with 95\% confidence intervals was also obtained. Sensitivity and specificity for each subscale were determined using ROC-determined cut-point scores.

\section{Participants' assessments}

A subset $(n=8)$ of the community participants had been queried in one-on-one interviews about the SDS-CL-17. Interviewees ranged from $33-73$ years old, were $50 \%$ female, $75 \%$ Cauca- sian, with educations from high school through post-bachelor's degree; half of the interviewees were diagnosed with OSA and half with insomnia during the community volunteer study. A pre-set list of questions was asked about the SDS-CL-17 regarding impressions of response options, item wording, and ease of completion. Interview notes of participants' responses were explored for key findings.

\section{Data management and statistics}

All data were provided for use in this study as SPSS data files. The data files as received included no personally identifiable information and were stored on a secure server at SUNY Buffalo. SPSS version 22 (SPSS Inc., Chicago, IL, USA) was used for all statistical analyses.

\section{RESULTS}

\section{Sample Characteristics}

The total number of subjects used for this validation study was $n=694$ (Table 1). The overall sample was $56 \%$ female averaging 45 years old with average BMI of 31.7. The sleep clinic pa- 
Table 1. Subject demographics, SDS-CL-17 subscale scores, and sleep disorder diagnoses

\begin{tabular}{|c|c|c|c|}
\hline Characteristic & $\begin{array}{l}\text { Community volunteers } \\
\qquad(\mathrm{n}=299)\end{array}$ & $\begin{array}{l}\text { Sleep clinic patients } \\
\qquad(\mathrm{n}=395)\end{array}$ & $\begin{array}{c}\text { Total } \\
(\mathrm{n}=694)\end{array}$ \\
\hline Age, mean (SD) & $40(17)$ & $48(13)$ & $45(16)$ \\
\hline Sex (proportion female) & $68 \%$ & $48 \%$ & $56 \%$ \\
\hline Body mass index, mean (SD) & $27(6.0)$ & $35(8.5)$ & $31.7(8.5)$ \\
\hline Epworth Sleepiness Scale, mean (SD) & $6.9(4.8)$ & $11.2(5.3)$ & $9.3(5.5)$ \\
\hline \multicolumn{4}{|l|}{ SDS-CL subscale scores, mean (SD) } \\
\hline Obstructive sleep apnea & $5.5(2.6)$ & $9.3(3.5)$ & $7.8(3.7)$ \\
\hline Insomnia & $7.5(3.1)$ & $9.3(3.5)$ & $8.5(3.5)$ \\
\hline Circadian rhythm disorder & $2.2(1.6)$ & $2.3(1.7)$ & $2.2(1.6)$ \\
\hline Restless legs syndrome & $3.3(2.3)$ & $4.9(3.3)$ & $4.2(3.0)$ \\
\hline Narcolepsy & $0.9(1.3)$ & $2.0(1.7)$ & $1.5(1.6)$ \\
\hline Parasomnias & $2.0(2.0)$ & $2.8(2.6)$ & $2.5(2.4)$ \\
\hline $\begin{array}{l}\text { Diagnosed with obstructive sleep apnea, } \\
\text { proportion (numbers) }\end{array}$ & $\begin{array}{c}27 \% \\
(\mathrm{n}=77 \mathrm{dx}, 283 \text { valid })\end{array}$ & $\begin{array}{c}85 \% \\
(\mathrm{n}=334 \mathrm{dx}, 395 \text { valid })\end{array}$ & $61 \%$ \\
\hline $\begin{array}{l}\text { Diagnosed with chronic insomnia disorder, } \\
\text { proportion (numbers) }\end{array}$ & $\begin{array}{c}24 \% \\
(\mathrm{n}=163 \mathrm{dx}, 298 \text { valid })\end{array}$ & $\begin{array}{c}55 \% \\
(\mathrm{n}=299 \mathrm{dx}, 395 \text { valid })\end{array}$ & $37 \%$ \\
\hline $\begin{array}{l}\text { Dual dx obstructive sleep apnea and insomnia, } \\
\text { proportion (numbers) }\end{array}$ & $\begin{array}{c}14 \% \\
(\mathrm{n}=39 \mathrm{dx}, 283 \text { valid })\end{array}$ & $\begin{array}{c}14 \% \\
(\mathrm{n}=56 \mathrm{dx}, 395 \text { valid })\end{array}$ & $14 \%$ \\
\hline $\begin{array}{l}\text { Diagnosed with circadian rhythm sleep-wake disorder, } \\
\text { proportion (numbers) }\end{array}$ & N/A & $0 \%$ & - \\
\hline $\begin{array}{l}\text { Diagnosed with restless legs syndrome, } \\
\text { proportion (numbers) }\end{array}$ & N/A & $\begin{array}{c}7 \% \\
(\mathrm{n}=28,395 \text { valid })\end{array}$ & - \\
\hline Diagnosed with narcolepsy, proportion (numbers) & $\mathrm{N} / \mathrm{A}$ & $\begin{array}{c}2 \% \\
(\mathrm{n}=8,395 \text { valid })\end{array}$ & - \\
\hline Diagnosed with parasomnias, proportion (numbers) & $\mathrm{N} / \mathrm{A}$ & $0 \%$ & - \\
\hline
\end{tabular}

dx: diagnosed, N/A: not available or not assessed, SD: standard deviation, SDS-CL-17: Sleep Disorders Symptom Checklist-17.

tients were significantly heavier (BMI of 35 vs. $27, \mathrm{p}<0.001$ ) and reported more daytime sleepiness (ESS of 11.2 vs. $6.9, \mathrm{p}<0.001$ ) in comparison to the community volunteers. This is not unexpected, as the sleep clinic patients had been referred to the sleep clinic for confirmatory diagnosis of sleep disorder(s), some of which (e.g., OSA) tend to be more prevalent as BMI increases. Similarly, patients referred to sleep clinics would be expected to report higher levels of daytime sleepiness, on average, in comparison to a sample from the community at large.

\section{SDS-CL-17 Scores}

As can be seen in Table 1, the SDS-CL-17 subscale scores indicate what might be expected for the two samples: the sleep clinic patients, on average, scored higher on all six sleep disorder subscales than the community sample.

\section{Expert Diagnoses}

Among all study participants, none were diagnosed with either circadian rhythm sleep-wake disorders or parasomnias. OSA was the most prevalent sleep disorder in the total sample $(61 \%)$ as well as for the community volunteers (27\%) and sleep clinic referrals (85\%). The next most prevalent diagnosis was insomnia (37\% overall, $24 \%$ of community volunteers, $55 \%$ of sleep clinic patients). Interestingly, $14 \%$ of all study subjects received dual diagnoses of both OSA and insomnia. Among the sleep clinic participants, $7 \%$ were diagnosed with RLS and $2 \%$ with narcolepsy.

\section{Receiver Operator Characteristic Curve Determined Cut-Points}

Cut-point scores (above which a positive screen is indicated) were: 5 for insomnia disorder, 3 for OSA, 3 for RLS/PLMD, and 1 for narcolepsy. Using these cut-point scores, sensitivity/specificity/AUC (95\% confidence intervals) for each subscale were: 1) $0.70 / 0.64 / 0.72(0.69-0.76)$ for insomnia disorder, 2$)$ $0.74 / 0.67 / 0.76(0.72-0.80)$ for OSA, 3) 0.75/0.80/0.88 (0.830.93 ) for RLS/PLMD, and 4) $0.88 / 0.68 / 0.79$ (0.65-0.93) for narcolepsy.

\section{Participants' Assessments}

Overall, study participants endorsed use of the SDS-CL-17. Instructions were reported to be easily understood and read- 
able. Although only community volunteers participated in interviews, they were diagnosed with either insomnia or OSA, therefore their collective viewpoints are expected to be no different than those of the sleep clinic group. About three-fourths of the interviewees commented that remembering the past year was difficult, while others liked the idea of reporting symptoms over the last year because it was "a leveler" and allowed them to report how they usually were, without having to worry about a recent week or month being abnormal. In addition, those who reported the last year was difficult to remember indicated that their SDS-CL-17 responses represented "Whatever I'm usually like."

All the interviewed subjects stated they liked having frequencies or timeframes along with the descriptive words for response options, and that the choices seemed clear. All stated that the frequencies helped them choose how to answer. One person commented on the once a year option, "Why bother, it's so close to never is it worth mentioning?"

\section{DISCUSSION}

Given the strength of the SDS-CL-17 validation study, the 17 -item instrument could be used in practice with the cutpoints and scoring algorithms as described above. Patients who complete the SDS-CL-17 and yield any sleep disorder subscale score above its respective cut-point (i.e., 5 for insomnia disorder, 3 for OSA, 3 for RLS/PLMD, and 1 for narcolepsy) would warrant follow-up as described in the "Follow-up to Screening Results" section below. However, while the SDS-CL-17 is a useful instrument, continued experience with this version indicated that several changes were required to make the questionnaire more comprehensive, more clinically relevant, and more informative.

First, a document header was created to gather patient characteristics. The header requests the patient's age, sex, height, and weight. Such data provide convergent evidence with respect to some of the diagnoses (e.g., OSA is more likely to occur in males who are older adults and overweight). The header also queries about work shift, number of hours worked per week, whether the respondent has a bed partner, what the typical sleep period is (from what time to what time), how many hours of sleep the patient typically gets per night, and how many hours the patient typically spends in bed each night. The question regarding shift work is to determine if the sleep complaints (as endorsed in items 1-6 and 25) may be, in part or in total, related to "shift work sleep disorder."

Information about weekly work hours allows a means to assess "sleep opportunity." Knowing whether the patient co-sleeps identifies a potential corroborator regarding the data provided and the possibility that a bed partner may be a contributory factor. Typical sleep period is used to assess the subject's preferred sleep phase, and total sleep time has a variety of purposes including evaluation of sleep duration (whether this is within normal limits or represents a problem in and of itself) and indirect evidence for the phenomenon of sleep state misperception. Time in bed, in combination with sleep duration, provides a rough estimation of sleep efficiency and the potential need to assess further for insomnia symptoms and/or the subject's appropriateness for cognitive behavior therapy for insomnia.

Second, the response time frame was altered from "the past year" to the "last 3 months." This is not only consistent with contemporary classifications of chronic sleep disorders, but may also allow for easier, and potentially more accurate, patient responses.

Third, the rating scale was changed to be more sensitive to diagnostically relevant symptom frequencies. Specifically, the scale was shifted to refine ratings on a per-week basis and limit the per-month options; response options of " $1-3$ times per week", "3-5 times per week", and "> 5 times per week" were added, while "monthly" and "never" were retained. The three weekly frequencies allow differentiation between mild, moderate, and severe levels of symptomatology and the latter two frequencies represent the threshold for what is likely of clinical relevance.

Fourth, several questions were simplified. For example, one of the questions pertaining to narcolepsy was changed from "I have experienced sudden muscle weakness when laughing, joking, angry or during other intense emotions" to "When angered, humored, frightened, I experience sudden muscle weakness."

Lastly, eight questions were added, thus the SDS-CL-25 designation. Three of these questions, while not specific to any one diagnosis, provide information about sleep insufficiency, nightto-night variability sleep timing, and whether the endorsed sleep disturbance items are perceived as being related to daytime dysfunction. Two questions were added to enhance sensitivity to OSA, two to assess for narcolepsy disorder, and one to assay for bruxism.

\section{The SDS-CL-25 Instrument}

The SDS-CL-25 can be administered (by paper or online) as a single standard page instrument $(8.5 \times 11$, one side only) and forms a grid of responses. Table 2 summarizes the SDS-CL-25 item and header content. The header captures patient data as described above. Below the header is a list of 25 symptom statements (at the fifth-grade Flesh-Kincaid reading level). Five response options are laid out as columns, so that patients can quickly scan and endorse with a "check mark" the relevant response ("never", "once a month", "1-3 times a week", "3-5 times a week", and "> 5 times a week"). Each row contains a single query and rows are clustered together to represent sleep disorders. Table 3 shows how the items are associated with the sleep disorders and functional outcomes being assessed instructions 
Table 2. Item content of the SDS-CL-25

\begin{tabular}{cl}
\hline Item & \\
\hline Header & Age, sex, height, weight \\
& H1: work shift \\
& H2: hours worked per week \\
& H3: presence of bed partner \\
& H4: amount of sleep each night \\
& H5: amount of time in bed each night \\
Q1 & Work/activity interferes w/sufficient sleep \\
Q2 & Bed or wake time variability \\
Q3 & Time to fall asleep \\
Q4 & Time awake during sleep period \\
Q5 & Early awakening \\
Q6 & Daytime sleepiness or fatigue \\
Q7 & Prefers early bed \& wake times \\
Q8 & Prefers late bed \& wake times \\
Q9 & Inappropriately falling asleep \\
Q10 & Snores \\
Q11 & Morning dry mouth \\
Q12 & Snoring interferes w/others sleep \\
Q13 & Stops breathing while asleep \\
Q14 & Gasps while asleep \\
Q15 & Leg sensations \\
Q16 & Urge to move legs at night \\
Q17 & Frequent nighttime awakenings for unknown reason \\
Q18 & Muscle weakness w/strong emotions \\
Q19 & Frightening images as awakens \\
Q20 & Can't move as awakens \\
Q21 & Nightmares \\
Q25 & Strange uehaviors while sleeping \\
&
\end{tabular}

SDS-CL-25: Sleep Disorders Symptom Checklist-25.

are to respond regarding how frequently the statements apply over the past three months.

In evolving to its current 25-item version, the instrument's depth and breadth grew beyond what might be expected by adding a short header and eight items. The SDS-CL-25 assesses for thirteen sleep disorders and four functional outcomes of sleep. The thirteen sleep disorders include insomnia (initial, middle, and late), advanced sleep phase syndrome (ASPS), delayed sleep phase syndrome (DSPS), OSA, RLS/PLMD, narcolepsy, nightmare disorder, night terror disorder, REM sleep behavior disorder, sleep-related TMJ, sleep insufficiency disorder, shiftwork sleep disorder, EDS not otherwise specified. The four functional outcomes of sleep include night-to-night variability of sleep, daytime dysfunction, and fatigue.

\section{SDS-CL-25 Interpretation}

At present the SDS-CL-25 does not have a validated scoring algorithm, this work is in progress (see future directions). This said, the interpretation method delineated in Table 3 represents a common sense approach to determining: 1) the likelihood of an individual having one or more of the 13 assessed sleep disorders, 2) the severity of presenting complaint by category, and 3) a method for assessing cumulative morbidity. With respect to the identification of one or more individual sleep disorders, any symptom grouping with at least one clinically relevant symptom (i.e., occurring 3 or more times a week) can be interpreted as a positive screen requiring follow up. With respect to relative severity of the individual sleep disorders, one may simply sum the individual item scores for each category and divide by the number of items to yield an average score per disorder. With respect to cumulative morbidity, one may simply sum all of the endorsements across the 25 items, with a resulting range of 0 to 100 . It is anticipated that the ongoing validation studies will yield a similar strategy but it is likely that individual items will not be equally weighted.

This approach is very sensitive and likely much more liberal in terms of screen positives than the SDS-CL-17. For example, an endorsement of snoring on 3 or more days per week is suggestive and consistent with the diagnosis of OSA but may nevertheless result in a false positive. This is consistent with the use of the SDS-CL-25 as a screening instrument and underscores the need for additional assessment. This said, endorsement of more than one symptom per category may increase the clinician's confidence that the patient may have one or more of the core sleep disorders. Header information can also be used to aid interpretation of the responses. For example, if the SDSCL-25 score for OSA is just below the suggested threshold, but the patient is an overweight male, the provider may decide that follow-up is warranted.

Deconstructing the parasomnias subscale is also recommended, with further exploration of these symptoms, perhaps by discussion or referral, for patients who endorse any one of the individual items 20-24. Nightmare disorder is represented by item 21 , night terrors by item 22 , REM sleep-behavior disorder by items 20 and 23, and sleep-related TMJ by item 24 .

For all patients, sleep duration (header), interference of activities with achieving sufficient sleep (item 1), night-to-night variability of sleep (item 2), daytime dysfunction (item 25), and EDS and/or fatigue (item 6) warrant exploration for potential follow-up. Simple assessment of sleep duration (header) determines whether amount of sleep might be an issue. For example, those who indicate typical sleep durations of 5.5 hours but claim to have no daytime consequences (items 6 and 25) may be naturally short sleepers. Information regarding presence of a bed partner (header data) or activities that interfere with sleep 
Table 3. SDS-CL-25 interpretation approach

\begin{tabular}{|c|c|}
\hline Assessment & Items to consider* \\
\hline \multirow[t]{2}{*}{ Insomnia disorder } & $(\mathrm{Q} 3$ or $\mathrm{Q} 4$ or $\mathrm{Q} 5)+(\mathrm{Q} 6$ or $\mathrm{Q} 9$ or $\mathrm{Q} 25)$ \\
\hline & Q17 may also be highly endorsed \\
\hline Advanced sleep phase syndrome & $\mathrm{Q} 7+\mathrm{Q} 5$ + (Q6 or Q9 or Q25) \\
\hline Delayed sleep phase syndrome & $\mathrm{Q} 8+\mathrm{Q} 3+(\mathrm{Q} 6$ or Q9 or Q25) \\
\hline Obstructive sleep apnea & Q10 - Q14 + (Q6 or Q9 or Q25) \\
\hline Restless legs syndrome/periodic limb movement disorder & Q15 - Q17 + (Q6 or Q9 or Q25) \\
\hline Narcolepsy & Q18 - Q20 + Q9 (also Q6 or Q25) \\
\hline Nightmare disorder & $\mathrm{Q} 21+\mathrm{Q} 25$ \\
\hline Night terror disorder & Q22 \\
\hline REM sleep behavior disorder & Q23 \\
\hline Sleep-related TMJ & Q24 \\
\hline Sleep insufficiency disorder & H4 and H5 + Q1 + (Q6 or Q9 or Q25) \\
\hline Shiftwork sleep disorder & $\mathrm{H} 1+(\mathrm{Q} 1$ or $\mathrm{Q} 2$ or Q3) or $(\mathrm{H} 4$ and/or Q1) + (Q6 or Q9 or Q25) \\
\hline Excessive daytime sleepiness non-specific & Q9 \\
\hline Fatigue & Q6 \\
\hline Total sleep time (TST) & $\mathrm{H} 4$ \\
\hline Time in bed (TIB) & H5 \\
\hline Sleep efficiency (\%) & $100 \times \mathrm{TST} / \mathrm{TIB}=100 \times \mathrm{H} 4 / \mathrm{H} 5$ \\
\hline Shift worked & H1 \\
\hline Informant & H3-can verify Q10, Q12-15, Q23 \\
\hline Normal short sleep variant & H4 without (Q6 or Q9 or Q25) \\
\hline General concern & (Q6 or Q9 or Q25) without other Q's endorsed \\
\hline
\end{tabular}

*Interpretation strategy: each Q is scored as follows: 0 points for a response of "never", 1 point for a response of "once a month", 2 points for a response of "1-3 times a week", 3 points for a response of " $3-5$ times a week", 4 points for a response of "> 5 times a week". Identification of individual sleep disorders: disorder may be deemed appropriate for follow-up if one or more endorsements within the category has response option 3 or 4 checked. Severity of the individual sleep disorders: sum the individual item scores for each category and divide by the number of items to yield an average score per disorder. Cumulative morbidity: sum all of the endorsements across the 25 items.

TMJ: temporomandibular joint disorder, SDS-CL-25: Sleep Disorders Symptom Checklist-25.

(item 1) can help determine if patients might achieve more sleep by managing lifestyle choices. Individuals whose work/ life activities interfere with sufficient sleep (item 1) may need more frequent and/or vigilant consultations to follow how short sleep may be impacting their overall cardiovascular or mental health.

\section{Follow-Up to Screening Results}

For patients who screen positive for one or more sleep disorder, follow-up could include treatment in-house by the primary care provider, referral to a specialist and/or follow-up with validated single-disorder questionnaires such as the ISI for insomnia disorder (7 items), ${ }^{13}$ the STOP for OSA (4 items), ${ }^{17}$ the Ullanlinna Narcolepsy Scale (11 items) for narcolepsy disorder ${ }^{18}$ and the Munich parasomnias questionnaire (21 items) for parasomnia disorders. ${ }^{19}$ Positive screens from these single-disorder questionnaires can be used to bolster the SDS-CL-25's suggestion for follow-up.

Unfortunately, no single or multi disorder questionnaire ex- ists that covers ASPS, DSPS, and/or shift work related sleep disorder. For DSPS, the best possible follow-up inquiry is to determine whether the individual's sleep schedule varies significantly from weekdays to weekends (or from work/school nights to days-off). Patients' responses to items 1 and 2 of the SDS-CL-25 can inform this determination. Yet, there is no hard-and-fast rule about what constitutes a significant deviation. The best rule-of-thumb is that schedules discordant by 3 or more hours suggest DSPS. In contrast, ASPS while often occurs as a stable invariant shift towards early time-to-bed and early morning awakenings. Here again, there is not a standard metric, but a shift of 3 or more hours (SDS-CL-25 item 7) suggests ASPS. It is possible to assess preferred sleep phases although not specific circadian rhythm sleep-wake disorders using the Morningness Eveningness Questionnaire ${ }^{20}$ or the Munich Chronotype Questionnaire, ${ }^{21}$ which provide self-report measures of patients' chronotypes.

Finally, the International Restless Legs Scale ${ }^{22}$ assesses for RLS, this instrument is solely a metric for illness severity and pre- 
sumes that the patient knows they have this particular disorder. Further, there is not a subscale for the signs and symptoms of PLMD. This may be the case because RLS is virtually pathognomonic for PLMD. This said, PLMD frequently can and does occur in the absence of RLS and thus likely warrants separate or independent assessment. Self-report assessment of PLMD, however, is nearly impossible and usually requires polysomnographic assessment for detection. The clinician may suspect PLMD when the patient reports pathological daytime sleepiness (e.g., strong endorsement of SDS-CL-25 item 9 regarding falling asleep at inappropriate times or places) in the absence of other sleep fragmentation related complaints.

Treating in-house may depend on providers' knowledge and comfort level with the individual sleep disorders. Referrals to specialists for further assessment and/or treatment may occur by collaborating with local sleep disorders centers and sleep medicine specialists, by collaborating with behavioral sleep medicine specialists who specialize in the behavioral treatment of sleep disorders, using treatments such as cognitive behavioral therapy for insomnia or positional therapy for sleep apnea. In the event that local resources are unknown, several provider directories are available: http://www.sleepeducation.org/finda-facility, http://www.behavioralsleep.org/ (select "provider search"), and http://www.med.upenn.edu/cbti/provder_directory.html.

\section{Future Directions}

Presently, SDS-CL-25 data are being collected as part of a study to establish a database comprising polysomnography, physician diagnoses, and sleep-related questionnaire responses. The study is expected to include over $n=1000$ adult subjects representative of primary care patients. Analysis of these data will enable: 1) validation of the SDS-CL-25 as a sleep disorders screener against gold standard physician and polysomnographic diagnoses, 2) convergent validity of the SDS-CL-25 with validated single disorder instruments, 3) convergent validity of the SDS-CL-25 with the one other brief global screener (the GSAQ), 4) verification that the SDS-CL-25 can be used to characterize cumulative sleep disorders morbidity, and 5) evaluation of whether the SDS-CL-25 is sensitive to treatment-related change. This data set will allow for convergent validity analysis, assessment of stability over time, and additional psychometric assessments of the new instrument.

\section{Concluding Remarks}

A notable advantage of the SDS-CL-25 is that visually scanning a single sheet of paper affords a quick and thorough assessment of the likely presence or absence of each of the assessed sleep disorders. Response patterns with marks to the right side of the grid, i.e., symptom endorsements for 3 or more days per week, indicate likely presence of sleep disorder(s) and/or need for follow-up.
In closing, the SDS-CL-25 is available on line at https://redcap.upstate.edu/surveys/?s=DNT8PL7PNA (at time of manuscript submission, the website is a functional beta-test version). The on-line version, upon completion, may be printed. Alternatively, a PDF version of the instrument may be downloaded from the same site. Clinical practice use of the instrument does not require permission. Research use of the instrument requires permission from the development team and may be arranged by contacting the first author of this report. Updated versions of the SDS-CL-25, use information, and newly available data regarding the psychometric properties of the instrument will also be available at the instrument website noted above.

\section{Conflicts of Interest}

The authors have no financial conflicts of interest.

\section{REFERENCES}

1. Gallicchio L, Kalesan B. Sleep duration and mortality: a systematic review and meta-analysis. J Sleep Res 2009;18:148-58.

2. Liu Y, Wheaton AG, Chapman DP, Croft JB. Sleep duration and chronic diseases among U.S. adults age 45 years and older: evidence from the 2010 behavioral risk factor surveillance system. Sleep 2013;36:1421-7.

3. Institute of Medicine, Board on Health Sciences Policy, Committee on Sleep Medicine and Research. Functional and economic impact of sleep loss and sleep-related disorders. In: Colten HR, Altevogt BM. Sleep disorders and sleep deprivation: an unmet public health problem. 1st ed. Washington, DC: National Academies Press (US) 2006;137-72.

4. World Health Organization. Global surveillance, prevention and control of chronic respiratory diseases: a comprehensive approach. Geneva: World Health Organization 2007.

5. Institute of Medicine, Board on Health Sciences Polic, Committee on Sleep Medicine and Research. Sleep disorders and sleep deprivation: an unmet public health problem. Washington, DC: National Academies Press (US) 2006.

6. Kroenke K, Spitzer RL. The PHQ-9: a new depression diagnostic and severity measure. Psychiatr Ann 2002;32:509-15.

7. Kroenke K, Spitzer RL, Williams JB. The PHQ-9: validity of a brief depression severity measure. J Gen Intern Med 2001;16:606-13.

8. Klingman KJ, Jungquist CR, Perlis ML. Questionnaires that screen for multiple sleep disorders. Sleep Med Rev 2017;32:37-44.

9. Roth T, Zammit G, Kushida C, Doghramji K, Mathias SD, Wong JM, et al. A new questionnaire to detect sleep disorders. Sleep Med 2002;3: 99-108.

10. Jungquist CR, Marcus JA, Valerio TD, White TV, Perlis ML, Pigeon $\mathrm{WR}$, et al. Validation of the sleep disorders screening questionnaire. Sleep 2011;34(Abstract Supplement):A329.

11. Jungquist CR, Mund J, Aquilina AT, Klingman K, Pender J, Ochs-Balcom $\mathrm{H}$, et al. Validation of the behavioral risk factor surveillance system sleep questions. J Clin Sleep Med 2016;12:301-10.

12. Johns MW. Sleepiness in different situations measured by the Epworth Sleepiness Scale. Sleep 1994;17:703-10.

13. Morin CM, Belleville G, Bélanger L, Ivers H. The Insomnia Severity Index: psychometric indicators to detect insomnia cases and evaluate treatment response. Sleep 2011;34:601-8.

14. American Psychiatric Association. Diagnostic and Statistical Manual of Mental Disorders: DSM-IV. 4th ed. Washington, DC: American Psychiatric Association 2000.

15. Streiner DL, Norman GR. Health measurement scales: a practical guide to their development and use. 4th ed. Oxford: Oxford University Press 2008.

16. Hanley JA, McNeil BJ. The meaning and use of the area under a receiver operating characteristic (ROC) curve. Radiology 1982;143:29-36. 
17. Chung F, Yegneswaran B, Liao P, Chung SA, Vairavanathan S, Islam S, et al. STOP questionnaire: a tool to screen patients for obstructive sleep apnea. Anesthesiology 2008;108:812-21.

18. Hublin C, Kaprio J, Partinen M, Koskenvuo M, Heikkilä K. The Ullanlinna Narcolepsy Scale: validation of a measure of symptoms in the narcoleptic syndrome. J Sleep Res 1994;3:52-9.

19. Fulda S, Hornyak M, Müller K, Cerny L, Beitinger PA, Wetter TC. Development and validation of the Munich Parasomnia Screening (MUPS). Somnologie (Berl) 2008;12:56-65.
20. Horne JA, Ostberg O. A self-assessment questionnaire to determine morningness-eveningness in human circadian rhythms. Int $\mathrm{J}$ Chronobiol 1976;4:97-110.

21. Roenneberg T, Wirz-Justice A, Merrow M. Life between clocks: daily temporal patterns of human chronotypes. J Biol Rhythms 2003;18:80-90.

22. Walters AS, LeBrocq C, Dhar A, Hening W, Rosen R, Allen RP, et al. Validation of the International Restless Legs Syndrome Study Group rating scale for restless legs syndrome. Sleep Med 2003;4:121-32. 